\title{
Gentamicin Reduces Calcific Nodule Formation by Aortic Valve Interstitial Cells In Vitro
}

\author{
Aditya Kumar ${ }^{\star}$, Dena C. Wiltz ${ }^{*}$, and K. Jane Grande-Allen \\ Department of Bioengineering, MS 142, Rice University, 6100 Main Street, Houston, TX 77005, \\ USA
}

\section{Abstract}

Purpose-Gentamicin is a widely employed antibiotic, but may reduce calcium uptake by eukaryotic cells. This study was conducted to determine whether gentamicin reduces calcification by porcine aortic valvular interstitial cells (pAVICs) grown in 2D culture, which is a common model for calcific aortic valve disease (CAVD).

Methods and Results-The presence of gentamicin (up to $0.2 \mathrm{mM}$ ) in the medium of pAVICs cultured for 8 days significantly lowered calcification and alkaline phosphatase content in a dosedependent manner compared to pAVICs cultured without gentamicin. Gentamicin also significantly increased cell proliferation and apoptosis at concentrations of 0.1-0.2 mM. Next, gentamicin was applied to previously calcified pAVIC cultures (grown for 8 days) to determine whether it could stop or reverse the calcification process. Daily application of gentamicin for 8 additional days significantly reduced calcification to below the pre-calcification levels.

Conclusions-These results confirm that gentamicin should be used cautiously with in vitro studies of calcification, and suggest that gentamicin may have the ability to reverse calcification by pAVICs. Given the nephrotoxicity and ototoxicity of this antibiotic, its clinical potential for the treatment of calcification in heart valves is limited. However, further investigation of the pathways through which gentamicin alters calcium uptake by valvular cells may provide insight into novel therapies for CAVD.

\section{Keywords}

gentamicin; calcification; mitochondrial calcium uptake; cell culture

\section{INTRODUCTION}

The use of antibiotics can cause unintended side effects that alter cellular behavior. One such antibiotic whose side effects have warranted investigation is gentamicin, an aminoglycoside antibiotic that is widely used in vitro to prevent cell and tissue culture contamination. ${ }^{1}$ Numerous in vitro studies have shown, however, that gentamicin can affect lipid levels and calcium uptake in cells. ${ }^{2-6}$ For example, both porcine kidney epithelial and

\footnotetext{
Address for correspondence: K. Jane Grande-Allen, PhD, Department of Bioengineering, Rice University, 6100 Main St., MS 142, Houston, TX 77005, USA, Phone: 713-348-3704, Fax: 713-348-5877, grande@ rice.edu.

These authors contributed equally to this work
} 
rabbit proximal tubular cells treated with gentamicin showed dose-dependent increases in the content of phospholipids, ${ }^{2,3}$ which aid in initiation and regulation of calcium hydroxyapatite formation in normal and pathological mineralization. ${ }^{7,8}$ Gentamicin has also been shown to reduce mitochondrial calcium uptake, block calcium channels, or otherwise compete with calcium binding dose-dependently in kidney and liver cells, ${ }^{4,9}$ nerve terminals ${ }^{5}$ and cardiac and vascular tissue. ${ }^{6}$ Thus, if gentamicin is used as antimicrobial agent in studies in which the mechanisms of calcification are being investigated, the results may be inadvertently influenced by the gentamicin.

One such area that is garnering attention regarding the mechanisms of calcification is calcific aortic valve disease (CAVD). CAVD is a degenerative disease with a prevalence of $2-3 \%$ in persons over the age of 75 years, ${ }^{10}$ and is the second most common indication for cardiac surgery. ${ }^{11}$ CAVD was originally believed to be due to passive accumulation of hydroxyapatite mineral in thickened, sclerotic valves, but recent evidence indicates an active cellular process in calcific remodeling. ${ }^{12}$ Though the identification of potential therapies has been hampered by a limited number of animal models that faithfully replicate the key histological features of CAVD, ${ }^{13}$ numerous studies have employed in vitro 2-D culture of valvular interstitial cells (VICs) to generate "calcific" nodules that stain positively for lipids, calcium, and phosphate. ${ }^{14}$ These in vitro nodules demonstrate certain constituents found in human calcified valves, including alkaline phosphatase, osteopontin, bone morphogenetic protein 2, and matrix Gla protein. ${ }^{14,15}$ Indeed, analysis of nodules produced by aortic VICs cultured with pathological stimuli, such as cholesterol or angiotensin peptides, has delineated several aspects of the complex mechanism underlying CAVD development. ${ }^{11,14}$ Additional in vitro studies investigating other regulatory pathways associated with CAVD have demonstrated the influence of RhoA and Rho kinase, Wnt/LRP5, Notch1, and a multitude of additional pathways on nodule formation by porcine VICs. ${ }^{16-18}$ Although this $2 \mathrm{D}$ cell culture model is simplistic, ${ }^{14}$ the analysis of in vitro nodule formation has proven to be a useful tool in the study of the mechanisms of CAVD.

Although it does not appear that gentamicin has been previously employed in investigations of CAVD in vitro, the reported effects of gentamicin on calcium uptake in other cell types offer a compelling motivation to examine its effects on VIC cultures, especially in studies of mineralization. The nephrotoxicity and ototoxicity associated with gentamicin may limit its clinical use. ${ }^{19,20}$ Nonetheless, investigating the effect of gentamicin on VICs would complement the growing interest in the role of mitochondrial calcium uptake on the progression of CAVD, especially regarding altered oxidative stress and the production of superoxides, ${ }^{21,22}$ which recruit inflammatory cells and likely contribute to extracellular matrix remodeling and calcification. ${ }^{23}$ Thus, the development of a therapeutic solution that targets mitochondrial calcium uptake could potentially curb the progression of CAVD.

Therefore, the purpose of this research was to assess the effect of gentamicin on the formation of calcific nodules by porcine aortic VICs (pAVICs) in vitro. Calcification was evaluated by staining for calcium and phosphate, the primary constituents of mineralized valvular calcium deposits, as well as by quantifying the enzyme alkaline phosphatase, which can cause the release of inorganic phosphate. ${ }^{24}$ Alkaline phosphatase plays a key role in the formation of the bone-like mineralization found in CAVD and the genes regulating alkaline 
phosphatase production are among the first expressed in the process of calcification. ${ }^{24,25}$ Two different studies were performed. First, to investigate the ability of gentamicin to prevent calcification, pAVICs were grown in gentamicin concentrations ranging from $0 \mathrm{mM}$ to $0.2 \mathrm{mM}$; the standard working concentration is $0.1 \mathrm{mM}$ according to manufacturer's instructions. Second, to investigate the ability to halt or reverse calcification, calcified pAVIC cultures were generated and then treated with gentamicin during varied time periods.

\section{METHODS}

\section{Cell Isolation and Culture}

Porcine hearts from healthy 6-month-old pigs were obtained from a local commercial abattoir (Fisher Ham and Meats, Spring Cypress, TX). Valves from multiple hearts were pooled together (between 2 and 5 hearts per cell harvest) and used to isolate pAVICs according to a two-step collagenase digestion as previously described. ${ }^{26}$ The isolation of primary cells was performed on at least two different occasions. pAVICs were passaged twice (P2) before experimentation.

\section{Effects of Gentamicin on Cellular Mineralization, Proliferation, and Apoptosis}

To determine the effects of gentamicin on the production of calcific nodules by pAVICs, P2 cells were plated in 24-well or 48-well plates at a density of $50,000 \mathrm{cells} / \mathrm{cm}^{2}$ and cultured in Dulbecco's Modification of Eagle's Medium (DMEM containing $1 \mathrm{~g} / \mathrm{L}$ glucose, Mediatech Inc., Manassas, VA) with Ham's F-12 nutrient mixture (50:50, Hyclone, Logan, UT), 1.6\% HEPES (Hyclone), 1\% antibiotic-antimycotic (ABAM, Mediatech) and 1\% bovine growth serum (BGS, Mediatech). The media also contained either 0 (control group), 0.01, 0.05, 0.1, or $0.2 \mathrm{mM}$ gentamicin (Mediatech). The cells were cultured for 8 days, with medium changes every 48 hours.

To determine whether gentamicin affects pAVIC proliferation, a repeat of the 8 day gentamicin concentration study was performed. On day 8, cell number in each well was determined via the Methylthiazol Tetrazolium (MTT) assay. A $50 \mu \mathrm{L}$ volume of MTT (5 $\mathrm{mg} / \mathrm{ml}$ in dI $\mathrm{H}_{2} \mathrm{O}$, Sigma-Aldrich, Saint Louis, MO) was added directly to $500 \mu \mathrm{L}$ of $1 \%$ BGS media in each well of a 24 -well plate and incubated at $37^{\circ} \mathrm{C}$ for 4 hours. The media and MTT reagent were removed, and $500 \mu \mathrm{L}$ of $0.1 \mathrm{~N} \mathrm{HCl}$ in anhydrous isopropanol was then added. $100 \mu \mathrm{L}$ of the solution was transferred to a 96-well plate. MTT content was determined by measuring absorbance at 570 and $690 \mathrm{~nm}$ and subtracting the latter from the former (Spectramax M2, Molecular Devices, Sunnyvale, CA).

To determine whether gentamicin affects pAVIC apoptosis, which can contribute to calcific nodule formation by valve cells, ${ }^{27}$ another repeat of the 8 day gentamicin study was performed. To assess the content of two effector caspases involved in the regulation of apoptosis, ${ }^{28}$ caspases 3 and 7, the Caspase-Glo 3/7 Assay Kit (Promega, Madison, WI) was used according to manufacturer's instructions. Caspase content was normalized to DNA content, which was measured using the Quant-iT Picogreen dsDNA Kit (Invitrogen, Eugene, OR). On day 8 , cells were lifted with $0.25 \%$ trypsin-EDTA (Mediatech) at $37^{\circ} \mathrm{C}$ for 5 minutes, pelleted and resuspended in $1 \mathrm{~mL}$ of $10 \%$ BGS media. Volumes of $100 \mu \mathrm{L}$ of the 
resuspended cells were mixed with $100 \mu \mathrm{L}$ of Caspase-Glo reagent containing picogreen (the picogreen reagent was added directly to the Caspase-Glo reagent at a ratio of 1:200) in wells of a 96-well plate and incubated at room temperature for 30 minutes. After incubation, the content of caspases 3 and 7 was determined by measuring luminescence (Spectramax M2). Next, the wells were incubated for an additional 30 minutes at room temperature and DNA content was measured by exciting the samples at $480 \mathrm{~nm}$ and measuring emissions at $520 \mathrm{~nm}$ (Spectramax M2).

\section{Effects of Gentamicin on Previously Calcified Cell Cultures}

To test the ability of gentamicin to reverse or stop calcification, a 16 day study was conducted. P2 pAVICs were plated as described above and cultured for 8 days in DMEM:F12 (1\% ABAM, 1\% BGS) containing $10 \mathrm{mM} \beta$-glycerophosphate (SigmaAldrich), with changes of medium every other day. $\beta$-glycerophosphate is a substrate for alkaline phosphate, ${ }^{29}$ which has been associated with CAVD progression, ${ }^{30}$ and has been shown to accelerate calcific nodule formation in cultures of bovine vascular smooth muscle cells. ${ }^{29,31}$ Here, $\beta$-glycerophosphate was added to generate strongly calcified pAVIC cultures as a starting point for the second half of the study. Starting on day 8 , the $\beta$ glycerophosphate treatment was discontinued and gentamicin $(0.2 \mathrm{mM})$ was added in different application patterns, with daily changes of medium (Table I). These different application patterns consisted of treatments for either $0,1,4$, or 8 days. The single (1) treatment was on day 8 . There were 3 versions of the 4 day gentamicin treatments: one day on - one day off (pattern performed 4 times), two days on - two days off (pattern performed twice), or four days on - four days off (performed one time).

\section{Calcium Detection}

At the conclusion of the study (day 8 or day 16), the pAVIC cultures were fixed with $10 \%$ formalin $\left(500 \mu \mathrm{L}\right.$ per well) and cells were stored overnight at $4^{\circ} \mathrm{C}$. The formalin was removed the next day and each well was washed twice with phosphate buffered saline (PBS, $\mathrm{pH}$ 7.4). The calcium content of each well was assessed by adding $500 \mu \mathrm{L}$ of $40 \mathrm{mM}$ Alizarin Red S (ARS, MP Biomedicals, Solon, OH, pH 4.1-4.3) and gently agitating for 30 minutes on a reciprocal shaker (Lab-Line Instruments, Melrose Park, IL) at 125 oscillations/ min. Excess stain was then removed by washing twice with PBS. All of the formed calcific nodules in each well were counted and imaged using an inverted microscope (DMIL, Leica Microsystems Inc., Buffalo Grove, IL) and 10X images of nodules were captured using a charge coupled device (CCD) camera (DFC 320, Leica). To assess calcium content histologically, the areas of the calcific nodules were measured from the images using outlining and measuring tools in ImageJ (NIH, Bethesda, MD). Total calcified area was calculated by summing the areas of nodules for each well.

\section{Phosphate and Alkaline Phosphatase Detection}

In preparation for measurements of phosphate, the pAVIC cultures on the final study days were fixed with $10 \%$ formalin and stored overnight as described above. The formalin was removed the next day and each well was washed twice with $500 \mu \mathrm{L}$ of $\mathrm{ddH}_{2} \mathrm{O}$. The phosphate content was demonstrated by the von Kossa stain. A $500 \mu \mathrm{L}$ volume of silver nitrate solution (Sigma, $1 \%$ in $\mathrm{ddH}_{2} \mathrm{O}$ ) was added to each well of the 24-well plate, which 
was then exposed to UV light (100W, UVP, LLC, Upland, CA) for 30 minutes. After removing the silver nitrate solution, the cells were washed with $\mathrm{ddH}_{2} \mathrm{O}$, and then incubated with $500 \mu \mathrm{L}$ of sodium thiosulfate solution (Fisher Scientific, Fairlawn, NJ; 5\% in $\mathrm{ddH}_{2} \mathrm{O}$ ) for $5 \mathrm{~min}$ at room temperature to stop the reaction. The sodium thiosulfate solution was removed and each well was washed twice with $\mathrm{ddH}_{2} \mathrm{O}$. Phosphate content was quantitatively assessed using the same nodule measurements described for the Alizarin Red S.

To assess alkaline phosphatase content, the SensoLyte FDP Alkaline Phosphatase Assay Kit (Anaspec Inc., Freemont, CA) was used according to manufacturer's instructions. On the final study days, the cell extract of each well in a 48-well plate was prepared by first removing the media and then adding $100 \mu \mathrm{L}$ of $0.2 \%$ Triton X-100 to each well. The extract from each well was then placed in a microcentrifuge tube and centrifuged for $10 \mathrm{~min}$ at $2500 \mathrm{~g}$. $50 \mu \mathrm{L}$ of the $100 \mu \mathrm{L}$ supernatant was removed and placed in a 96-well plate and 50 $\mu \mathrm{L}$ of 3,6-fluorescein diphosphate was added to each well and incubated for $30 \mathrm{~min}$ at $37^{\circ} \mathrm{C}$. The amount of alkaline phosphatase was determined by exciting the samples at $485 \mathrm{~nm}$ and measuring the emissions at $528 \mathrm{~nm}$.

\section{Statistics}

Each experiment was performed in two biological replicates, each with a sample size of 5 wells per gentamicin concentration or application pattern. Data were presented as mean \pm standard deviation of the combined 10 sample wells. Statistical comparisons among nodule number, nodule size, total calcified area, alkaline phosphatase, cell proliferation and cell apoptosis (caspases 3 and 7) were obtained using one-way ANOVA followed by Tukey post-hoc tests using the statistical analysis software JMP (SAS, Cary, NC). The level of significance was set at $a=0.05$.

\section{RESULTS}

\section{Effects of Gentamicin on Cellular Mineralization}

For the ARS assay, there was a significant reduction in the number of nodules, although a significant increase in the average size per nodule (representative nodules shown in Fig. 1ab), with every increasing concentration of gentamicin. The overall decrease in nodule number and increase in nodule size from the control group to the $0.2 \mathrm{mM}$ gentamicin group was $49 \%$ and $57 \%$, respectively (Fig. 2a-b). The total calcified area was significantly reduced with every increase in gentamicin concentration except the 0.1 and $0.2 \mathrm{mM}$ groups, resulting in $21 \%$ less calcified area at the highest gentamicin concentration of $0.2 \mathrm{mM}$ (Fig. 2c).

Similar to the ARS assay, the von Kossa staining showed a significant decrease in nodule number and a significant increase in nodule size at every increasing concentration of gentamicin (data not shown; representative nodules shown in Fig. 1c-d). The overall decrease in nodule number and increase in nodule size from the control group to the $0.2 \mathrm{mM}$ gentamicin group was $47 \%$ and $51 \%$, respectively. The total calcified area also decreased with gentamicin concentration, with the $0.2 \mathrm{mM}$ gentamicin group having $20 \%$ less calcified area than the control group (Fig. 3a). The same pattern was shown by the alkaline 
phosphatase assay, with the $0.2 \mathrm{mM}$ gentamicin group having $49 \%$ less alkaline phosphatase compared to control (Fig. 3b). For both von Kossa total calcified area and the alkaline phosphatase assay, all gentamicin concentration groups were significantly different from each other except for the 0 and $0.01 \mathrm{mM}$ gentamicin groups.

\section{Effect of Gentamicin of Cellular Proliferation and Apoptosis}

There was a significant increase in cell number for the $0.05,0.1$, and $0.2 \mathrm{mM}$ gentamicin groups compared to control ( $p<0.05$ ), with a $45 \%$ increase in cell number for the $0.2 \mathrm{mM}$ gentamicin group compared to control (Fig. 4a). Similarly, there was a significant increase in the content of caspases 3 and 7 for the $0.1 \mathrm{mM}$ and $0.2 \mathrm{mM}$ gentamicin groups, with a $70 \%$ increase in caspase content in the $0.2 \mathrm{mM}$ gentamicin group compared to control (Fig. $4 b)$.

\section{Effects of Gentamicin on Previously Calcified Cell Cultures}

There were two control groups for this experiment. The first control was the initial control group, which had been previously calcified by $\beta$-glycerophosphate treatment for 8 days. In addition, a final control 16 day group was included, in which the samples were allowed to calcify endogenously, without $\beta$-glycerophosphate or gentamicin, for an additional 8 days. By all measures, the final control group was significantly more calcified than the initial control group (Fig. 5-6).

The ARS assay showed that treatment every day with gentamicin reduced the number of nodules and total calcified area compared to the 16 day control. Interestingly, treatment every day reduced the total calcified area and nodule number to $27 \%$ and $31 \%$, respectively, less than the initial 8-day control (Fig. 5a-b). Treatment for only one day, in contrast, reduced nodule number below the final 16 day control but there was no difference in total calcified area between one day and the final 16 day control. The groups in which gentamicin was added for 4 days total (in different application patterns) were not significantly different from each other but all had lower nodule number and total calcified area compared to the final 16 day control values. The two groups that had gentamicin applied for 2 consecutive days both had fewer nodules than in the initial 8 day control. There was no effect of treatment pattern on average nodule size.

von Kossa staining showed results that were comparable to ARS (Fig. 6a-b). All gentamicin application patterns resulted in reduced nodule number and total calcified area compared to the 16 day control. Furthermore, gentamicin treatment every day reduced these values below the 8 day initial control (reduced $26 \%$ for nodule number and $32 \%$ for total calcified area). As with ARS, there was no significant difference between groups for nodule size. The values for nodule number and total calcified area in the every day gentamicin group were significantly lower than for all other groups $(p<0.05)$.

With respect to alkaline phosphatase, all treatments of 4 or 8 days total showed less content than the 16 day final control, and the content in the every day treatment group was lower than all 4 day groups (Fig. 6c). The every day treatment was not statistically different from the 8 day initial control. 


\section{DISCUSSION}

This study showed that gentamicin has the ability not only to reduce calcification in twodimensional pAVIC cultures but also reduce the calcification in cultures with pre-existing nodules. More specifically, it was observed that total calcification and alkaline phosphatase significantly decreased in a manner dependent upon the dose or frequency of application. There was also a significant increase in cell proliferation and apoptosis at the highest gentamicin concentrations. This work was the first to demonstrate the effects of gentamicin in valvular interstitial cell cultures. Based on research with other cell types, the ability of gentamicin to reduce calcification may be due to interference with $\mathrm{Ca}^{2+}$ uptake pathways, such as the calcium uniporter (MCU) on the mitochondrial inner membrane and the calcium channels and superficial binding sites on the plasma membrane. Because the application of gentamicin was also found to reduce calcification below pre-existing levels, calcium efflux pathways may have also been involved.

Our results from the 8 day study, showing that there was an overall reduction in the total amount of calcification in the pAVIC cultures treated with gentamicin, are consistent with published reports of reduced calcium uptake in similarly treated cultures of renal epithelial, myocardial, smooth muscle, and neural cells. ${ }^{2,4-6}$ Dystrophic calcification is characterized by increased calcium uptake in response to cellular injury; thus, reduction of calcium uptake could possibly curb the progression of pathological calcification. ${ }^{32,33}$ For example, mitochondrial calcium uptake decreased by $20 \%$ and $40 \%$ for rat renal cortical cells cultured in media containing 0.025 and $0.25 \mathrm{mM}$ gentamicin, respectively. ${ }^{4}$ The effect of aminoglycosides, such as gentamicin, on mitochondrial calcium uptake appears to be celltype dependent; gentamicin was shown to induce $\mathrm{Ca}^{2+}$ accumulation in rat liver mitochondria at concentrations below $500 \mu \mathrm{M}$, but reduced $\mathrm{Ca}^{2+}$ accumulation at higher concentrations. ${ }^{9}$ In a study of porcine kidney cells treated with $1 \mathrm{mM}$ gentamicin for 4 days, cell-associated $\mathrm{Ca}^{2+}$ content and $\mathrm{Ca}^{2+}$ uptake were reduced by approximately $30 \%$ and $20 \%$, respectively. ${ }^{2}$ In an organ culture study, cultured guinea pig inner ear tissue treated with 0.5 $\mathrm{mM}$ gentamicin had 30\% and 20\% lower amounts of bound calcium in the Organ of Corti and Stria Vascularis, respectively. ${ }^{34}$ Likewise, rat brush-border membrane cells prepared from homogenized renal cortex showed $70 \%$ and $80 \%$ reductions in calcium uptake when they were treated with $0.1 \mathrm{mM}$ and $1 \mathrm{mM}$ gentamicin, respectively. ${ }^{35}$ Other aminoglycoside antibiotics, such as neomycin, have also been shown to inhibit calcium uptake levels by vascular and neural cells. ${ }^{36,37}$

Alkaline phosphatase has also been reported to be affected by gentamicin treatment in various cell types. In one study, alkaline phosphatase production by human mesenchymal stem cells cultured in media containing gentamicin decreased in a dose-dependent manner. ${ }^{38}$ Alkaline phosphatase was similarly significantly reduced in cultures of human fetal osteoblast cells treated with $25 \mu \mathrm{g} / \mathrm{ml}(0.05 \mathrm{mM})$ gentamicin $^{39}$ and mouse myoblast $\mathrm{C} 2 \mathrm{C} 12$ cells cultured for 48 hours with gentamicin in concentrations of $12.5-800 \mu \mathrm{g} / \mathrm{ml}(0.025-1.6$ $\mathrm{mM}){ }^{40}$ The minimum dose of gentamicin required to affect calcification does appear to depend on the cell type; we found alkaline phosphatase to be significantly decreased in pAVIC cultures treated with gentamicin at concentrations greater than $25 \mu \mathrm{g} / \mathrm{mL}(0.05 \mathrm{mM})$, whereas another study of human osteoblastlike cells found that a significant decrease in 
alkaline phosphatase required treatment with gentamicin in concentrations of at least 100 $\mu \mathrm{g} / \mathrm{mL}(0.2 \mathrm{mM}) .^{41}$ However, those cells were only exposed to gentamicin for 4 days as opposed to 8 days in our study.

One mechanism through which gentamicin may inhibit uptake of calcium is through the mitochondrial calcium uniporter. Both gentamicin and magnesium are capable of inhibiting calcium uptake through the MCU, although not necessarily in a competitive manner. ${ }^{4,42}$ It is thought that gentamicin interferes with calcium uptake through the MCU in a manner similar to the inhibitory action of ruthenium red, an inorganic dye. Although ruthenium red does not directly bind to the calcium binding site, it inhibits mitochondrial calcium uptake by binding both to a region of high anionic density on the cytosolic side of the MCU and to adjacent negatively charged phospholipids. ${ }^{43}$ The binding of ruthenium red to these sites promote conformational changes in the MCU that modify the calcium activation site in such a way that the uptake cannot be activated. In the future, it will be important to assess calcium content in the mitochondria of valvular interstitial cells since this content is likely to play a key role in the development of pathological calcification. Dystrophic calcification, the primary form of calcification found in CAVD, first occurs due to increased influx of calcium into the mitochondria, which leads to the accumulation of calcium salt deposits that eventually spread throughout the cell. ${ }^{32,33}$ Additionally, increased mitochondrial $\mathrm{Ca}^{2+}$ content, which has been linked to CAVD, leads to mitochondrial oxidative stress, causing increased production of reactive oxidative species (ROS).${ }^{44}$ ROS such as hydrogen peroxide have been shown to accumulate in calcifying valve segments and promote the differentiation of vascular cells into vascular osteoblast-like cells, resulting in hydroxyapatite mineral formation. Furthermore, hydrogen peroxide is a pro-inflammatory secondary messenger responsible for the recruitment of inflammatory cells, the predominant cell type in early aortic valve lesions..$^{23,45}$ These cells release cytokines that contribute to extracellular matrix formation, remodeling, and local calcification. ${ }^{23}$ Thus, a means of preventing or reducing the influx of calcium into the mitochondria, usually caused by cell injury, could potentially curb the progression of dystrophic calcification.

Gentamicin has also been shown to reduce $\mathrm{Ca}^{2+}$ uptake into the cell by reducing the ability of cells to store and accumulate $\mathrm{Ca}^{2+}$. It is believed that aminoglycosides associate with anionic binding sites localized in membrane lipoproteins, resulting in the displacement of $\mathrm{Ca}^{2+}$ from superficial membrane sites that reduce the amount of $\mathrm{Ca}^{2+}$ available for transmembrane influx. ${ }^{46}$ Aminoglycosides also compete with $\mathrm{Ca}^{2+}$ for $\mathrm{Ca}^{2+}$ binding sites thought to be located at the outer orifice of $\mathrm{Ca}^{2+}$ channels. ${ }^{6}$ In addition, it has been demonstrated that aminoglycoside antibiotics can block stretch-receptor channels by reducing $\mathrm{Ca}^{2+}$ influx into muscle cells. A similar blockage of stretch-receptor channels in gentamicin-treated VICs would have a detrimental impact since mechanical stimulation is so essential to VIC function. ${ }^{47}$ Indeed, aminoglycoside antibiotics were shown to prevent mechanotransduction in murine outer hair cells, both by blocking the mechano-electrical transducer channels and by entering the cells through these channels. ${ }^{48,49}$ On the other hand, gentamicin is an agonist of the calcium-sensing receptor (CaSR), a G-protein coupled receptor that senses extracellular calcium concentration. Aminoglycoside-induced CaSR activation can result in increased intracellular $\mathrm{Ca}^{2+}$ accumulation and mobilization, ${ }^{50,51}$ although it has been shown for human aortic endothelial cells exposed to high extracellular 
$\mathrm{Ca}^{2+}$ that increased $\mathrm{Na}^{+} / \mathrm{Ca}^{2+}$ exchanger activity will counteract elevations in intracellular $\mathrm{Ca}^{2+}$ associated with CaSR activation, thus masking any bulk cytosolic response. ${ }^{52}$

In the second study, we found that daily gentamicin treatment of previously calcified cultures reduced calcification below the starting level. This finding suggests the involvement of mechanisms for calcium removal, as opposed to merely having reduced calcium uptake. Indeed, other studies have provided evidence for a role for gentamicin in calcium efflux; the application of gentamicin to rabbit aortic strips results in an increased rate of $\mathrm{Ca}^{2+}$ efflux. ${ }^{46,53}$ Cellular and mitochondrial calcium efflux is governed by $\mathrm{Na}^{+}$-dependent and $\mathrm{Na}^{+}$-independent mechanisms. ${ }^{54}$ Treatment with ruthenium red results in a slow but complete release of mitochondrial $\mathrm{Ca}^{2+}$, predominantly through the $\mathrm{Na}^{+} / \mathrm{Ca}^{2+}$ antiporter; 55 this antiporter is also thought to offset changes in cellular calcium content outside the mitochondria. In future studies, it will be of interest to evaluate the regulation of cytosolic and mitochondrial calcium uptake and efflux in investigations of valvular cell calcification.

The dose-dependent effects of gentamicin on nodule size and cell density, observed in the first study, may be due to the reported effects of gentamicin on cell proliferation and apoptosis, although these effects appear to be cell-type-specific. For example, cell number was significantly increased in human skin fibroblasts treated with $100 \mu \mathrm{g} / \mathrm{mL}$ gentamicin, ${ }^{56}$ although concentrations less than $100 \mu \mathrm{g} / \mathrm{ml}$ did not affect the growth rate of human mesenchymal stem cells. ${ }^{57} \mathrm{Schwartz}$ et al. have proposed that gentamicin promotes cell proliferation by reducing lipid degradation, resulting in an excess of lipids. ${ }^{2}$ For example, porcine kidney epithelial cells treated for 4 days with $2 \mathrm{mM}$ gentamicin had $240 \%$ greater content of phosphatidylcholine, a phospholipid constituent of cell membranes with key roles in cell replication ${ }^{58}$ and the mitogenic signaling of endothelial growth factor and other growth factors via production of 1,2-diacylglycerol. ${ }^{59}$ Furthermore, mesangial cells cultured in $10^{-5} \mathrm{M}$ gentamicin media exhibited not only increased proliferation but also simultaneously increased apoptosis. ${ }^{60}$ This effect may be due in part to the agonist activity of gentamicin towards the CaSR, as this can upregulate the extracellular regulated protein kinase (ERK), Akt, and p38 MAPK pathways, and thus also impact cell proliferation and apoptosis. ${ }^{51}$ Given that apoptosis of valvular interstitial cells is reported to be an early event in calcific nodule development in vitro, and that nodule formation and calcification can be decoupled, ${ }^{27}$ gentamicin-induced apoptotic pAVICs likely contributed to the dosedependent increase in nodule size observed in the first study, even though the overall amount of calcification was reduced by gentamicin.

Although analysis of nodules formed from 2-D cell cultures is widely used as a model of cell-mediated calcification, ${ }^{14}$ this approach may be limited in its replication of in vivo cellular activity. Another study limitation was that during the gentamicin application period of the second study, the media was changed daily instead of every other day as in the first study and in the first 8 days of the second study. Although this frequency of medium change would not affect the results of groups cultured for all 16 days, it complicates a direct comparison between the two studies. The method of generating the calcific nodules (endogenous vs. $\beta$-glycerophosphate) also differed between the two studies. Nonetheless, the effects of gentamicin treatment were consistent between studies in depending upon either concentration or frequency of application. 
In conclusion, this study of the effects of gentamicin on pAVIC calcific nodule formation in vitro has suggested novel directions, namely the regulation of cellular calcium uptake and efflux, for future investigations of the mechanisms of CAVD. Moreover, it is evident that gentamicin should be used very cautiously in in vitro studies of calcification. Given the toxic effects of gentamicin and related aminoglycoside antibiotics on kidney function and hearing, ${ }^{19,20}$ treatment with gentamicin is far from an ideal solution to CAVD. Admittedly, there are reports that the toxicity of gentamicin can be mitigated by supplements such as fish oil and olive oil ${ }^{61}$ or free radical scavengers such as caffeic acid phenethyl ester. ${ }^{62}$ At the present time, however, it seems that a more promising outcome of this line of research would be to develop a better understanding of the mechanism by which gentamicin reverses calcification in order to generate new treatments for this challenging and prevalent valve disease.

\section{Acknowledgments}

The authors thank Melissa McHale, Ph.D. for her assistance in preparation of the manuscript. This research was funded by National Institutes of Health grant \# R21HL104377.

\section{References}

1. Hahn FE, Sarre SG. Mechanism of action of gentamicin. J Infect Dis. 1969; 119(4):364-9. [PubMed: 4892389]

2. Schwertz D, Kreisberg J, Venkatachalam M. Gentamicin-induced alterations in pig kidney epithelial (LLC-PK1) cells in culture. J Pharmacol Exp Ther. 1986; 236(1):254-62. [PubMed: 3941396]

3. Ramsammy LS, Josepovitz C, Lane B, Kaloyanides GJ. Effect of gentamicin on phospholipid metabolism in cultured rabbit proximal tubular cells. Am J Physiol. 1989; 256(1):204-13.

4. Sastrasinh M, Weinbergl JM, Humes HD. The effect of gentamicin on calcium uptake by renal mitochondria. Life Sci. 1982; 30(26):2309-15. [PubMed: 6810049]

5. Parsons TD, Obaid AL, Salzberg BM. Aminoglycoside antibiotics block voltage-dependent calcium channels in intact vertebrate nerve terminals. J Gen Physiol. 1992; 99(4):491-504. [PubMed: 1317913]

6. Gotanda K, Yanagisawa T, Satoh K, Taira N. Are the cardiovascular effects of gentamicin similar to those of calcium antagonists? Japan J Pharmacol. 1988; 47:217-27. [PubMed: 3221528]

7. Demer L. Lipid hypothesis of cardiovascular calcification. Circulation. 1997; 95:297-8. [PubMed: 9008435]

8. Raggio CL, Boyan BD, Boskey AL. In vivo hydroxyapatite formation induced by lipids. J Bone Miner Res. 1986; 1(5):409-15. [PubMed: 3503556]

9. Rustenbeck I, Eggers G, Reiter H, Mu W. Polyamine modulation of mitochondrial calcium transport: I. stimulatory and inhibitory effects of aliphatic polyamines, aminoglucosides and other polyamine analogues on mitochondrial calcium uptake. Biochem Pharmacol. 1998; 56(98):977-85. [PubMed: 9776308]

10. Lindroos M, Kupari M, Heikkilä J, Tilvis R. Prevalence of aortic valve abnormalities in the elderly: an echocardiographic study of a random population sample. J Am Coll Cardiol. 1993; 21(5):1220-5. [PubMed: 8459080]

11. Grande-Allen KJ, Osman N, Ballinger ML, Dadlani H, Marasco S, Little PJ. Glycosaminoglycan synthesis and structure as targets for the prevention of calcific aortic valve disease. Cardiovasc Res. 2007; 76(1):19-28. [PubMed: 17560967]

12. Mohler IIIER. Mechanisms of aortic valve calcification. Am J Cardiol. 2004; 94(11):1396-402. [PubMed: 15566910]

13. O'Brien KD. Pathogenesis of calcific aortic valve disease: a disease process comes of age (and a good deal more). Arterioscler Thromb Vasc Biol. 2006; 26:1721-8. [PubMed: 16709942] 
14. Guerraty M, Mohler ER III. Models of aortic valve calcification. J Invest Med. 2007; 55(6):27883.

15. Rajamannan NM. Calcific aortic stenosis: lessons learned from experimental and clinical studies. Arterioscler Thromb Vasc Biol. 2009; 29(2):162-8. [PubMed: 19023094]

16. Gu X, Masters KS. Role of the rho pathway in regulating valvular interstitial cell phenotype and nodule formation. Am J Physiol Heart Circ Physiol. 2011; 300(2):H448-58. [PubMed: 21131478]

17. Rajamannan NM. Calcific aortic valve disease: cellular origins of valve calcification. Arterioscler Thromb Vasc Biol. 2011; 31(12):2777-8. [PubMed: 22096095]

18. Nigam V, Srivastava D. Notch1 represses osteogenic pathways in aortic valve cells. J Mol Cell Cardiol. 2009; 47(6):828-34. [PubMed: 19695258]

19. Takada A, Schacht J. Calcium antagonism and reversibility of gentamicin-induced loss of cochlear microphonics in the guinea pig. Hear Res. 1982; 8:179-86. [PubMed: 7142042]

20. Mingeot-Leclercq MP, Tulkens PM. Aminoglycosides: nephrotoxicity. Antimicrob Agents Chemother. 1999; 43(5):1003-12. [PubMed: 10223907]

21. Miller JD, Chu Y, Brooks RM, Richenbacher WE, Peña-Silva R, Heistad DD. Dysregulation of antioxidant mechanisms contributes to increased oxidative stress in calcific aortic valvular stenosis in humans. J Am Coll Cardiol. 2008; 52(10):843-50. [PubMed: 18755348]

22. Dedkova EN, Ji X, Lipsius SL, Blatter LA. Mitochondrial calcium uptake stimulates nitric oxide production in mitochondria of bovine vascular endothelial cells. Am J Physiol Cell Physiol. 2004; 286(2):406-15.

23. Freeman RV, Otto CM. Spectrum of calcific aortic valve disease: pathogenesis, disease progression, and treatment strategies. Circulation. 2005; 111(24):3316-26. [PubMed: 15967862]

24. Golub EE, Boesze-Battaglia K. The role of alkaline phosphatase in mineralization. Curr Opin Orthop. 2007; 18(5):444-8.

25. Pohjolainen V, Taskinen P, Soini Y, Rysä J, Ilves M, Juvonen T, Ruskoaho H, Leskinen H, Satta J. Noncollagenous bone matrix proteins as a part of calcific aortic valve disease regulation. Hum Pathol. 2008; 39(11):1695-701. [PubMed: 18701137]

26. Stephens EH, Carroll JL, Grande-Allen KJ. The use of collagenase III for the isolation of porcine aortic valvular interstitial cells: rationale and optimization. J Heart Valve Dis. 2007; 16(2):175-83. [PubMed: 17484468]

27. Jian B, Narula N, Li Q, Mohler ER III, Levy RJ, ERM. Progression of aortic valve stenosis: TGFbeta1 is present in calcified aortic valve cusps and promotes aortic valve interstitial cell calcification via apoptosis. Ann Thorac Surg. 2003; 75:457-65. [PubMed: 12607654]

28. Walsh JG, Cullen SP, Sheridan C, Lüthi AU, Gerner C, Martin SJ. Executioner caspase-3 and caspase-7 are functionally distinct proteases. PNAS. 2008; 105(35):12815-9. [PubMed: 18723680]

29. Shioi A, Nishizawa Y, Jono S, Koyama H, Hosoi M, Morii H. B-glycerophosphate accelerates calcification in cultured bovine vascular smooth muscle cell. Arterioscler Thromb Vasc Biol. 1995; 15(11):2003-9. [PubMed: 7583582]

30. Rodriguez CJ, Bartz TM, Longstreth WT, Kizer JR, Barasch E, Lloyd-Jones DM, Gottdiener JS. Association of annular calcification and aortic valve sclerosis with brain findings on magnetic resonance imaging in community dwelling older adults: the cardiovascular health study. J Am Coll Cardiol. 2011; 57(21):2172-80. [PubMed: 21596233]

31. Speer MY, Giachelli CM. Regulation of cardiovascular calcification. Cardiovasc Pathol. 2004; 13(2):63-70. [PubMed: 15033154]

32. Charalampos A. Role of mitochondria in calcification. Mitochondrial activity distribution in the epiphyseal plate and accumulation of calcium and phosphate ions by chondrocyte mitochondria. Biochem Biophys Res Commun. 1972; 46(5):1928-35. [PubMed: 4335571]

33. Zhao Y, Urganus AL, Spevak L, Shrestha S, Doty SB, Boskey AL, Pachman LM. Characterization of dystrophic calcification induced in mice by cardiotoxin. Calcif Tissue Int. 2009; 85(3):267-75. [PubMed: 19690791]

34. Schacht J. Biochemistry of neomycin ototoxicity. J Acoust Soc Am. 1976; 59(4):940-4. [PubMed: 177473] 
35. Somermeyer MG, Knauss TC, Weinberg JM, Humes HD. Characterization of $\mathrm{Ca} 2+$ transport in rat renal brush-border membranes and its modulation by phosphatidic acid. Biochem J. 1983; 214(1): 37-46. [PubMed: 6615471]

36. Ciceri P, Volpi E, Brenna I, Elli F, Borghi E, Brancaccio D, Cozzolino M. The combination of lanthanum chloride and the calcimimetic calindol delays the progression of vascular smooth muscle cells calcification. Biochem Biophys Res Commun. 2012; 418(4):770-3. [PubMed: 22310712]

37. Atchison WD, Adgate L, Beaman CM. Effects of antibiotics on uptake of calcium into isolated nerve terminals. J Pharmacol Exp Ther. 1988; 245(2):394-401. [PubMed: 3367298]

38. Chang Y, Goldberg VM, Caplan AI. Toxic effects of gentamicin on marrow-derived human mesenchymal stem cells. Clin Orthop. 2006; 45:2242-9.

39. Ince A, Schütze N, Hendrich C, Jakob F, Eulert J, Löhr JF. Effect of polyhexanide and gentamicin on human osteoblasts and endothelial cells. Swiss Med Wkly. 2007; 137:139-45. [PubMed: 17370154]

40. Ince A, Schütze N, Karl N, Löhr JF, Eulert J. Gentamicin negatively influenced osteogenic function in vitro. Int Orthop. 2007; 31(2):223-8. [PubMed: 16710734]

41. Isefuku S, Joyner CJ, Simpson AH. Gentamicin may have an adverse effect on osteogenesis. J Orthop Trauma. 2003; 17(3):212-6. [PubMed: 12621263]

42. Gunter TE, Sheu S. Characteristics and possible functions of mitochondrial Ca2+ transport mechanisms. Biochim Biophys Acta. 2009; 1787(11):1291-308. [PubMed: 19161975]

43. Zazueta C, Sosa-Torres ME, Correa F, Garza-Ortiz A. Inhibitory properties of ruthenium amine complexes on mitochondrial calcium uptake. J Bioenerg Biomembr. 1999; 31(6):551-7. [PubMed: 10682913]

44. Peng T, Jou M. Oxidative stress caused by mitochondrial calcium overload. Ann NY Acad Sci. 2010; 1201:183-8. [PubMed: 20649555]

45. Mody N, Parhami F, Sarafian T, Demer L. Oxidative stress modulates osteoblastic differentiation of vascular and bone cells. Free Radic Biol Med. 2001; 31(4):509-19. [PubMed: 11498284]

46. Weiss B, Richard H. Alterations by neomycin of $45 \mathrm{Ca}$ movements and contractile responses in vascular smooth muscle. J Pharmacol Exp Ther. 1974; 188(2):472-80. [PubMed: 4204349]

47. Yeung EW, Whitehead NP, Suchyna TM, Gottlieb Pa, Sachs F, Allen DG. Effects of stretchactivated channel blockers on [Ca2+]I and muscle damage in the mdx mouse. J Physiol. 2005; 562(Pt 2):367-80. [PubMed: 15528244]

48. Kawashima Y, Géléoc GSG, Kurima K, Labay V, Lelli A, Asai Y, Makishima T, Wu DK, Santina CCD, Holt JR, Griffith AJ. Mechanotransduction in mouse inner ear hair cells requires transmembrane channel-like genes. J Clin Invest. 2011; 121(12):4796-809. [PubMed: 22105175]

49. Marcotti W, van Netten SM, Kros CJ. The aminoglycoside antibiotic dihydrostreptomycin rapidly enters mouse outer hair cells through the mechano-electrical transducer channels. J Physiol. 2005; 567(Pt 2):505-21. [PubMed: 15994187]

50. Molostvov G, James S, Fletcher S, Bennett J, Lehnert H, Bland R, Zehnder D. Extracellular calcium-sensing receptor is functionally expressed in human artery. Am J Physiol Renal Physiol. 2007; 293(3):F946-55. [PubMed: 17537980]

51. Ward DT, Maldonado-Pérez D, Hollins L, Riccardi D. Aminoglycosides induce acute cell signaling and chronic cell death in renal cells that express the calcium-sensing receptor. J Am Soc Nephrol. 2005; 16(5):1236-44. [PubMed: 15800129]

52. Magno AL, Ward BK, Ratajczak T. The calcium-sensing receptor : a molecular perspective. Endocr Rev. 2011; 32(1):3-30. [PubMed: 20729338]

53. Adams HR, Goodman FR, Weiss GB. Alteration of contractile function and calcium ion movements in vascular smooth muscle by gentamicin and other aminoglycoside antibiotics. Antimicrob Agents Chemother. 1974; 5(6):640-6. [PubMed: 15825418]

54. Gunter TE, Pfeiffer DR. Mechanisms by which mitochondria transport calcium. Am J Physiol. 1990; 258(5):C755-86. [PubMed: 2185657]

55. Bernardi P. Mitochondrial transport of cations: channels, exchangers, and permeability transition. Physiol Rev. 1999; 79(4):1127-55. [PubMed: 10508231] 
56. Goetz IE, Moklebust R, Warren CJ. Effects of some antibiotics on the growth of human diploid skin fibroblasts in cell culture. In Vitro. 1979; 15(2):114-9. [PubMed: 457178]

57. Kagiwada H, Fukuchi T, Machida H, Yamashita K, Ohgushi H. Effect of gentamicin on growth and differentiation of human mesenchymal stem cells. J Toxicol Pathol. 2008; 21:61-7.

58. Tercé F, Brun H, Vance DE. Requirement of phosphatidylcholine for normal progression through the cell cycle in C3H/10T1/2 fibroblasts. J Lipid Res. 1994; 35(12):2130-42. [PubMed: 7897311]

59. Wright TM, Shin HS, Raben DM. Sustained increase in 1,2-diacylglycerol precedes dna synthesis in epidermal-growth-factor-stimulated fibroblasts. evidence for stimulated phosphatidylcholine hydrolysis. Biochem J. 1990; 267(2):501-7. [PubMed: 2159287]

60. Martínez-Salgado C, Eleno N, Morales AI, Pérez-Barriocanal F, Arévalo M, López-Novoa JM. Gentamicin treatment induces simultaneous mesangial proliferation and apoptosis in rats. Kidney Int. 2004; 65(6):2161-71. [PubMed: 15149329]

61. Rashid F, Kaleem M, Bano B. Comparative effect of olive oil and fish oil supplementation in combating gentamicin induced nephrotoxicity in rats. Indian Journal of Clinical Biochemistry. 2005; 20(1):109-14. [PubMed: 23105505]

62. Balakumar P, Rohilla A, Thangathirupathi A. Gentamicin-induced nephrotoxicity: do we have a promising therapeutic approach to blunt it? Pharmacol Res. 2010; 62(3):179-86. [PubMed: 20434560] 

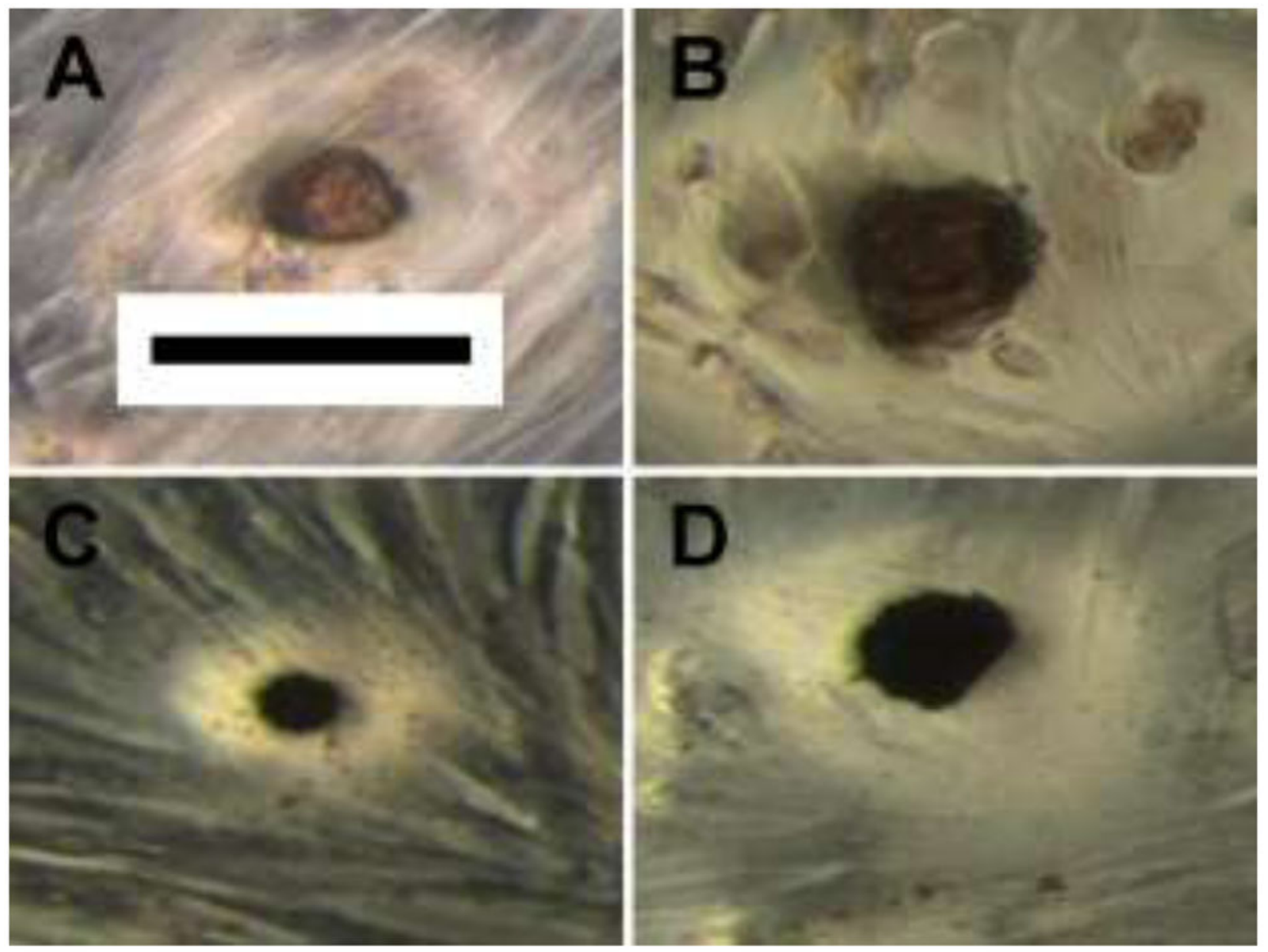

FIGURE 1.

Representative calcific nodules stained with Alizarin Red S (A, B) or Von Kossa (C, D). The cultures in images $\mathrm{A}$ and $\mathrm{C}$ were not treated with gentamicin whereas the cultures in images B and D were treated with $0.2 \mathrm{mM}$ gentamicin for 8 days (Scale bar is $100 \mu \mathrm{m}$ ) 
A Nodule Number-ARS

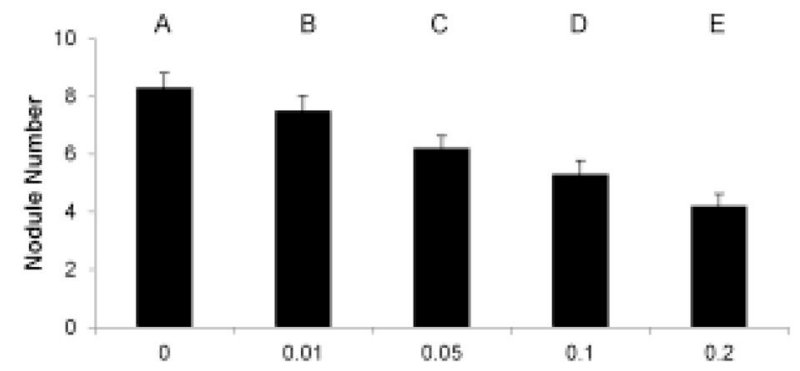

B

Nodule Size-ARS
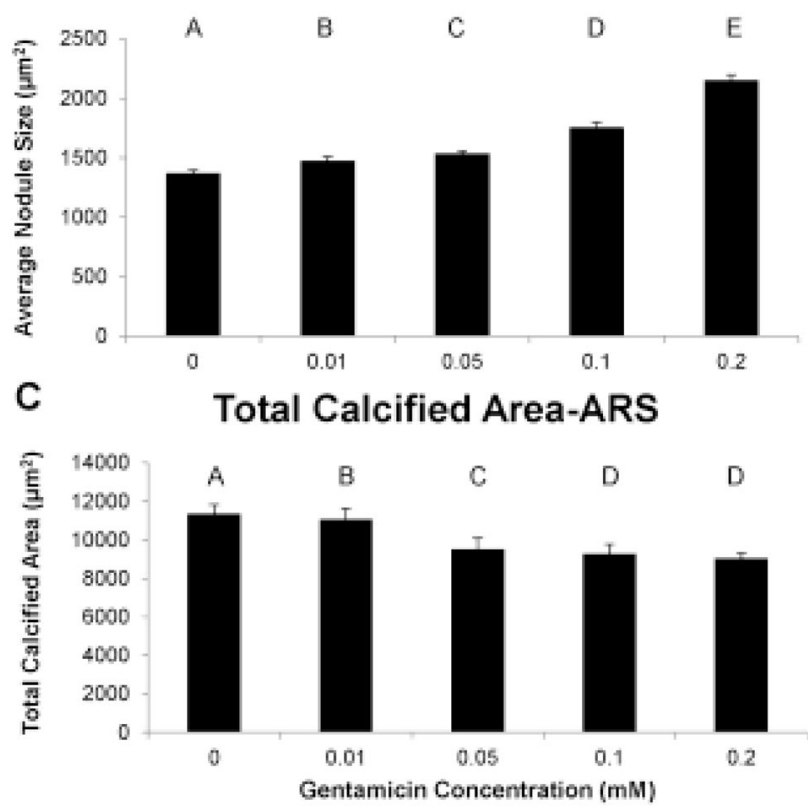

FIGURE 2.

Use of the Alizarin Red S stain showed that gentamicin concentration dose-dependently reduced nodule number $(\mathrm{A})$, increased nodule size (B), and reduced the total calcified area (C). Groups not connected by same letter are significantly different $(p<0.05)$ 


\section{A Total Calcified Area-Von Kossa}

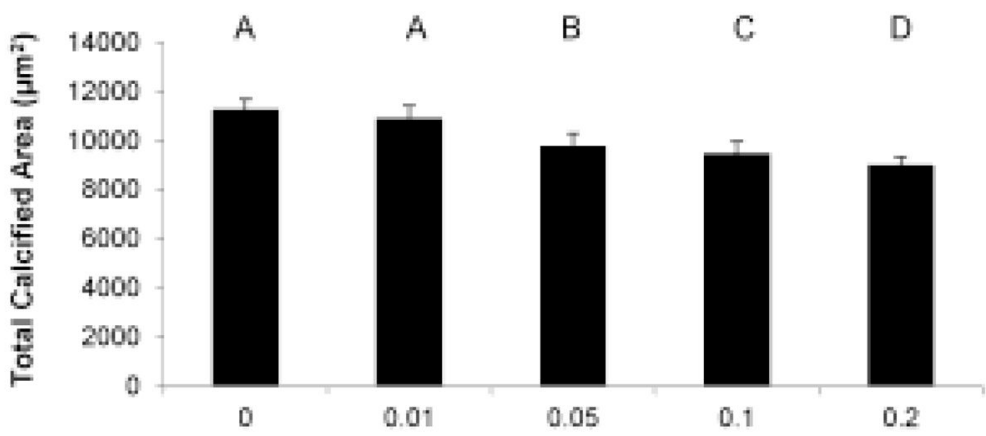

B

ALP Content

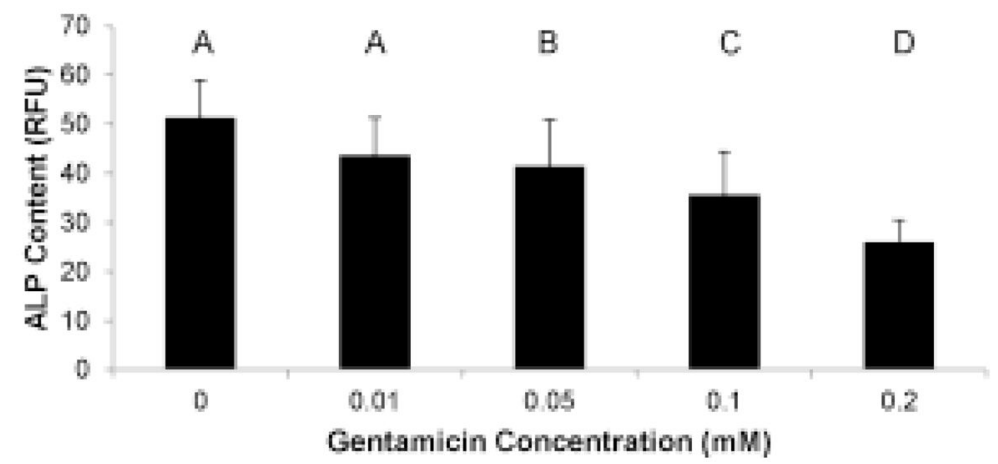

FIGURE 3.

Use of the von Kossa stain showed that gentamicin dose-dependently reduced the total calcified area (A) and alkaline phosphatase content (B) of the pAVIC cultures. Groups not connected by same letter are significantly different $(p<0.05)$ 

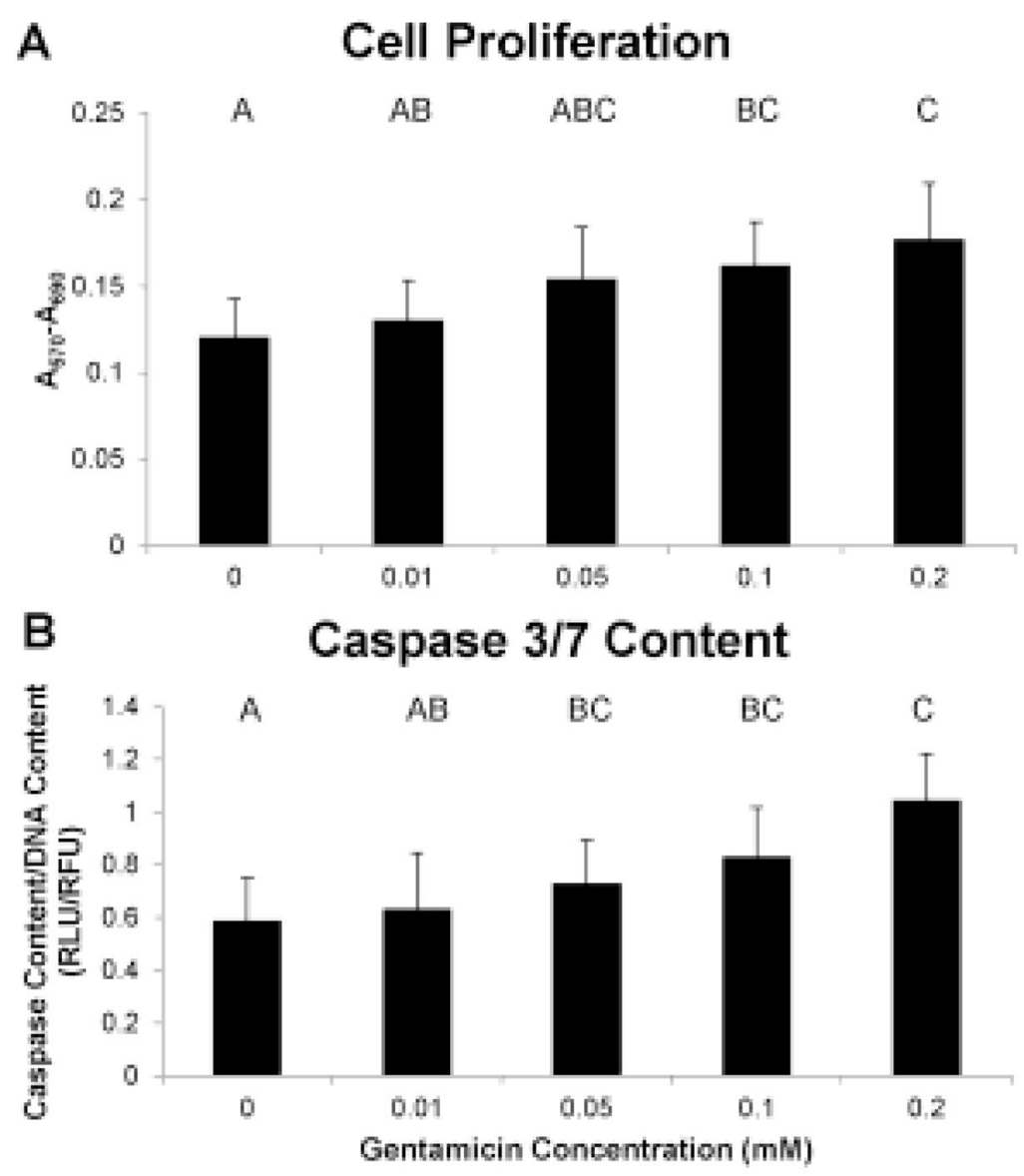

FIGURE 4.

Use of MTT assay and Caspase 3/7 assay showed that gentamicin dose-dependently increased cellular number (A) and apoptosis (B). Groups not connected by same letter are significantly different $(p<0.05)$ 

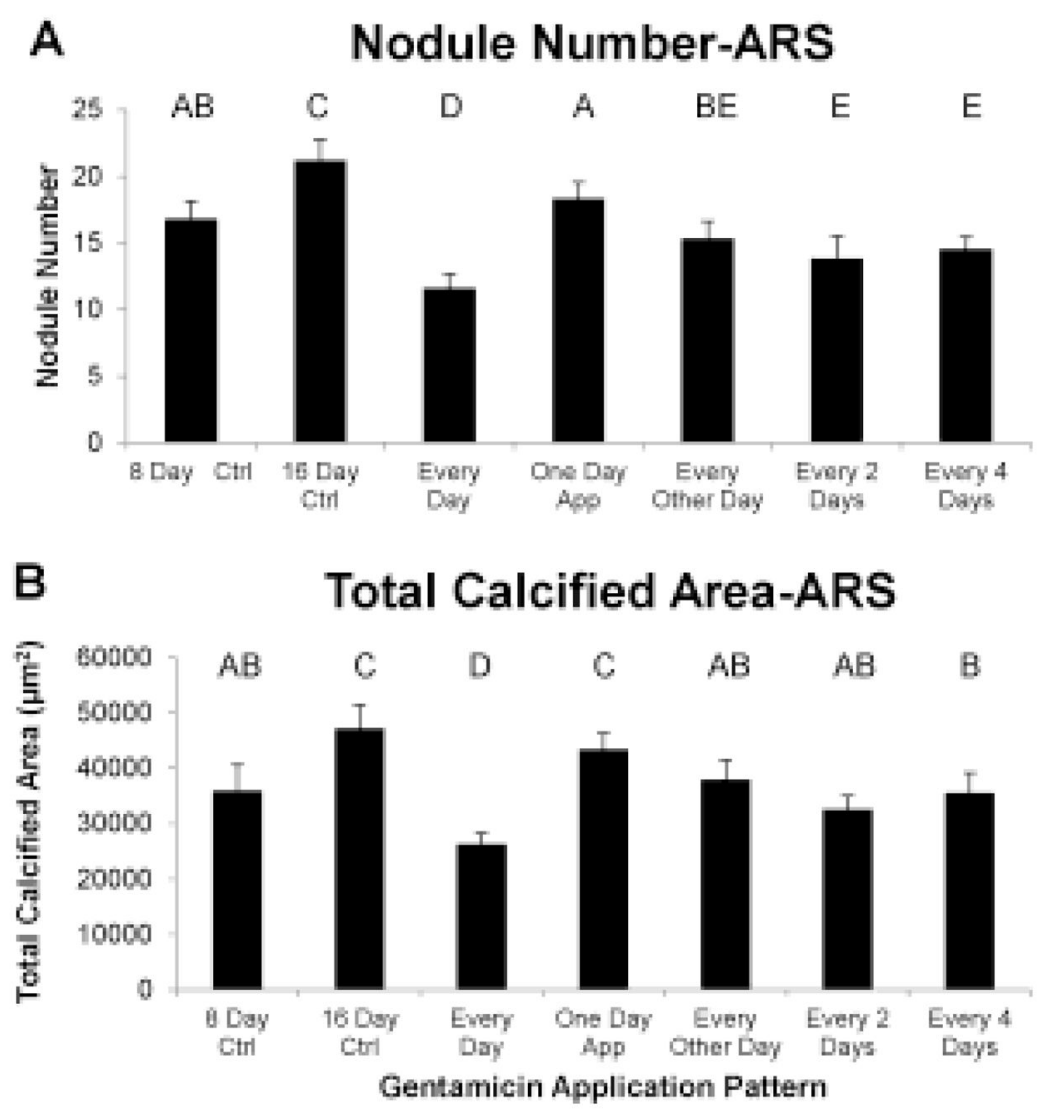

FIGURE 5.

The application of gentamicin to previously calcified pAVIC cultures reduced the number of calcium-containing nodules (A) and the total calcified area (B), as demonstrated by Alizarin Red S, depending upon the frequency of treatments. Groups not connected by same letter are significantly different 

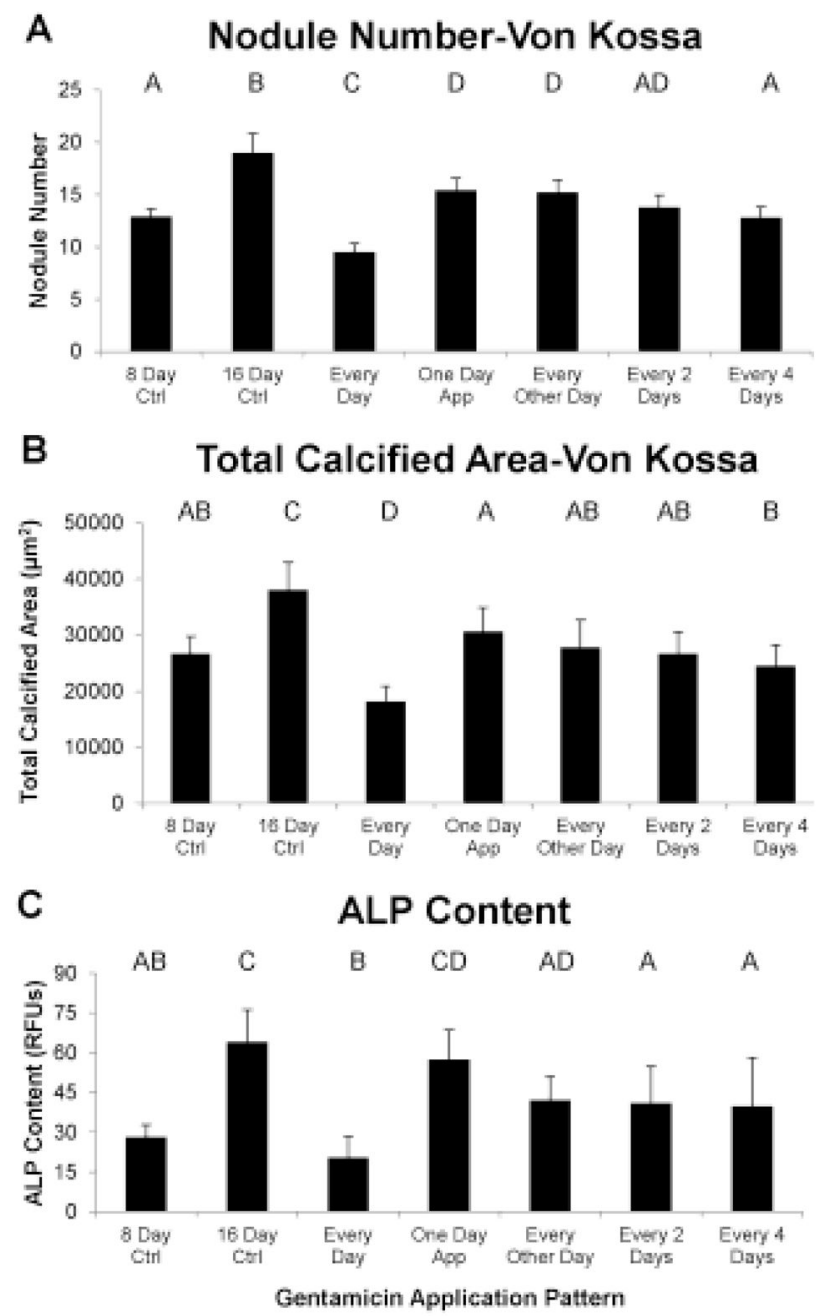

FIGURE 6.

The application of gentamicin to previously calcified pAVIC cultures reduced the number of phosphate-containing nodules (A) and the total phosphate nodule area (B), as demonstrated by von Kossa, as well as the content of alkaline phosphatase (C), depending upon the frequency of treatments. Groups not connected by same letter are significantly different 
